\title{
Searching for Inflow toward Massive Starless Clump Candidates Identified in the Bolocam Galactic Plane Survey
}

\author{
Jenny K. Calahan ${ }^{1}$, Yancy L. Shirley, Brian E. Svoboda, Elizabeth A. Ivanov, Jonathan R. Schmid, Anna Pulley, \\ Jennifer Lautenbach, Nicole Zawadzki, Christopher Bullivant, Claire W. Cook, Laurin Gray, Andrew Henrici, Massimo Pascale, \\ Carter Bosse, Quadry Chance, Sarah Choi, Marina Dunn, Ramon Jaime-Frias, Ian Kearsley, Joseph Kelledy, Collin Lewin, \\ Qasim Mahmood, Scott McKinley, Adriana M. Mitchell, and Daniel R. Robinson \\ Steward Observatory, University of Arizona, 933 North Cherry Avenue, Tucson, AZ 85721, USA; jcalahan@email.arizona.edu \\ Received 2018 January 3; revised 2018 April 19; accepted 2018 April 19; published 2018 July 23
}

\begin{abstract}
Recent Galactic plane surveys of dust continuum emission at long wavelengths have identified a population of dense, massive clumps with no evidence for ongoing star formation. These massive starless clump candidates are excellent sites to search for the initial phases of massive star formation before the feedback from massive star formation affects the clump. In this study, we search for the spectroscopic signature of inflowing gas toward starless clumps, some of which are massive enough to form a massive star. We observed 101 starless clump candidates identified in the Bolocam Galactic Plane Survey (BGPS) in $\mathrm{HCO}^{+} J=1-0$ using the $12 \mathrm{~m}$ Arizona Radio Observatory telescope. We find a small blue excess of $E=\left(N_{\text {blue }}-N_{\text {red }}\right) / N_{\text {total }}=0.03$ for the complete survey. We identified six clumps that are good candidates for inflow motion and used a radiative transfer model to calculate mass inflow rates that range from 500 to $2000 M_{\odot} \mathrm{Myr}^{-1}$. If the observed line profiles are indeed due to large-scale inflow motions, then these clumps will typically double their mass on a freefall time. Our survey finds that massive BGPS starless clump candidates with inflow signatures in $\mathrm{HCO}^{+} J=1-0$ are rare throughout our Galaxy.
\end{abstract}

Key words: ISM: clouds - ISM: molecules - ISM: structure - stars: formation

\section{Introduction}

Massive $\left(M>8 M_{\odot}\right)$ stars form from dense prestellar cores within massive molecular clumps (McKee \& Ostriker 2007). The theories of the dominant physical mechanisms responsible for their formation are still polemical (Motte et al. 2017). Do massive protostars form from the monolithic collapse of a massive $\left(M>30 M_{\odot}\right)$ prestellar core, or do protostellar cores accrete surrounding material and grow rapidly through a competitive accretion with the surrounding environment? This long-standing problem in the field of star formation can be addressed by looking for signatures of large-scale accretion, determining how often it occurs, and the rate at which material is accreting. In this paper, we present a spectroscopic survey of the optically thick, intermediate-density gas tracer, $\mathrm{HCO}^{+} 1-0$, searching for the signature of large-scale inflow in massive clumps in the Milky Way for which no signature of star formation has been previously detected.

Recent blind surveys of the Galactic plane at far-infrared through submillimeter wavelengths (i.e., BGPS, ATLASGAL, HiGAL, JPS; Ginsburg et al. 2013; Urquhart et al. 2014; Eden et al. 2017; Elia et al. 2017) have identified a population of tens of thousands of massive clumps $\left(M>200 M_{\odot}\right)$ that do not appear to be forming stars. These objects were classified as starless clump candidates (SCCs) in the evolutionary analysis of clumps identified in the $1.1 \mathrm{~mm}$ Bolocam Galactic Plane Survey (Svoboda et al. 2016). The typical SCC has a median mass of $230 M_{\odot}$, a size of $1 \mathrm{pc}$, a gas kinetic temperature (measured from $\mathrm{NH}_{3}$ observations) of $13 \mathrm{~K}$, and a starless phase lifetime that scales inversely with the mass of the clump (Svoboda et al. 2016). The clumps are starless candidates because current Galactic plane surveys are limited to detecting

\footnotetext{
${ }^{1}$ Senior Honors Thesis submitted at the University of Arizona.
}

protostars with luminosities $L>\sim 30 L_{\odot}$ at a distance of a few kiloparsecs (Svoboda et al. 2016). Even if there is a population of undetected low-mass protostars already forming in them, SCCs represent environments that are in an early stage of star formation and have not been severely disrupted by feedback from intermediate- or high-mass protostars (Matzner 2017). SCCs are ideal locations to search for evidence of large-scale flows that may be important in the formation of massive protostars.

A spectroscopic signature of inflow is the presence of a blue asymmetric, self-absorbed line profile, meaning that the line peaks at smaller velocities with respect to the $v_{\mathrm{LSR}}$ of the object and the line shape are double peaked with a clear self-abroption dip. The general conditions for the formation of this line profile are that the tracer must be optically thick enough to create selfabsorption with increasing excitation temperature and a favorable velocity field along the line of sight (i.e., the flow has a component projected along the line of sight). The presence of a blue asymmetric self-absorbed profile is not evidence of inflow alone, as other physical mechanics (namely, rotation and outflow) can also mimic similar line shapes under the appropriate conditions (see Evans 2003). Generally, a convincing case can be made when the spectral line profile is mapped over a core and radiative transfer models are used with independent constraints on the source physical structure, for instance, a density profile derived from dust continuum observations, to successfully model the observed line profile. Convincing collapse signatures seen in emission have been observed toward individual dense cores (e.g., Choi et al. 1995; Myers et al. 1995; Narayanan et al. 1998; Tafalla et al. 1998; Evans et al. 2005; Seo et al. 2011). Recently there have been observations of purported large-scale flows toward high-mass (Peretto et al. 2013), intermediate-mass (Kirk et al. 2013), and 


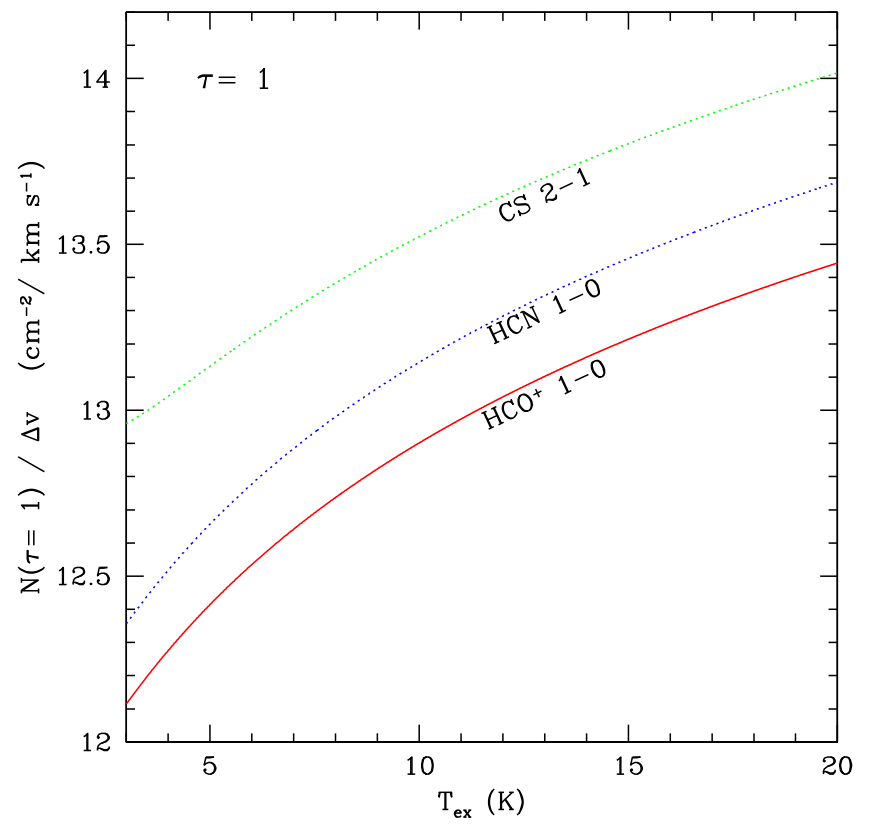

Figure 1. Column density at which a molecular tracer becomes optically thick (defined here as $\tau=1$ ) at the line peak plotted as a function of the excitation temperature. The curves were calculated assuming an FWHM line width of $\Delta v=1 \mathrm{~km} \mathrm{~s}^{-1}$. The HCN $1-0$ curve has not accounted for hyperfine structure; the curve for the strongest $\mathrm{HCN} 1-0$ hyperfine transition $(F=2-1)$ would be the plotted curve multiplied by $9 / 5=1.8$.

low-mass (Palmeirim et al. 2013) star-forming regions. A survey searching for the signature of inflow motions has not been performed yet toward the newly identified SCCs in the BGPS. A survey for inflow motions toward SCCs has the advantage that their kinematics should not be dominated by outflows since there is no detected star formation and therefore a blue asymmetric, self-absorbed profile is more likely to be associated with inflow.

For this survey, we chose the $\mathrm{HCO}^{+} 1-0$ line as an optically thick kinematic tracer of intermediate-density gas in SCCs. $\mathrm{HCO}^{+} 1-0$ has an effective excitation density of $\sim 1000 \mathrm{~cm}^{-3}$ (Shirley 2015). We can assess the column density at which a particular molecular tracer becomes optically thick using

$$
\frac{N_{\tau=1}\left(T_{\mathrm{ex}}\right)}{\Delta v}=4 \sqrt{\frac{\pi^{3}}{\ln 2}} \frac{\nu_{u l}^{3}}{g_{u} A_{u l} c^{3}} \frac{Q\left(T_{\mathrm{ex}}\right)}{e^{-E_{l} / k T_{\mathrm{ex}}}-e^{-E_{u} / k T_{\mathrm{ex}}}},
$$

where $T_{\mathrm{ex}}$ is the excitation temperature of the rotational transition from $u \rightarrow l$ with energy levels $E_{u}$ and $E_{l}$, frequency $\nu_{u l}$, statistical weight $g_{u}$, and Einstein $A_{u l} . Q\left(T_{\mathrm{ex}}\right)$ is the partition function assuming that every energy level has the same $T_{\text {ex }}$ (the CTEX approximation; see Mangum \& Shirley 2015). The threshold optically thick column density $N_{\tau=1}$ is a function of $T_{\mathrm{ex}}$ and the FWHM line width ( $\Delta v$ given in $\mathrm{cm} \mathrm{s}^{-1}$ ) where we have assumed a Gaussian line profile. Figure 1 shows these threshold column densities for commonly observed dense gas tracers assuming an FWHM line width of $1 \mathrm{~km} \mathrm{~s}^{-1}$. Typical $\mathrm{HCO}^{+}$column densities observed toward clumps in Galactic surveys are in the range of $10^{13}-10^{15} \mathrm{~cm}^{-2}$ (Hoq et al. 2013; Shirley et al. 2013), which is well above the threshold column density curve for all excitation temperatures $T_{\mathrm{ex}}<12 \mathrm{~K}$. $\mathrm{HCO}^{+}$ $1-0$ is an excellent optically thick tracer of intermediate-density, subthermally populated gas with which to search for the

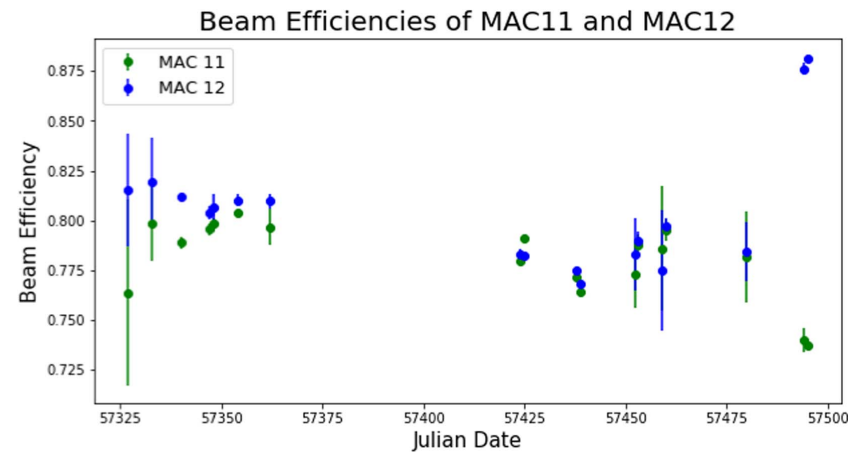

Figure 2. Main-beam efficiencies derived each day for each polarization (MAC 11 and MAC 12) and used for that day's data reduction. For MAC 11 the median value is 0.787 , and for MAC 12 it is 0.801 .

kinematic signatures of inflow. Simulations of global collapse agree with this assertion and further illustrate that the $1-0$ transition is the best transition with which to search for inflow signatures (Smith et al. 2013).

In this paper, we present a systematic survey of 101 SCCs located toward the first quadrant of the Milky Way in $\mathrm{HCO}^{+} 1-0$ using the $12 \mathrm{~m}$ Arizona Radio Observatory telescope. We present the observations (Section 2) and analyze the line profiles searching the signature of inflow (Section 3). We find six inflow candidates and model their line profiles using the HILL5 radiative transfer model (Section 4). Finally, we calculate mass inflow rates and discuss how this may affect the evolution of these clumps (Section 4).

\section{Observations}

We observed at the Arizona Radio Observatory (ARO) $12 \mathrm{~m}$ radio telescope on Kitt Peak for 19 observing shifts between 2015 September and 2016 April. $^{2}$ We tuned to the $J=1-0$ transition of $\mathrm{HCO}^{+}$at $89,188.5250 \mathrm{MHz}$ in the lower sideband using the ALMA Band 3 ARO prototype dual-polarization sideband-separating receiver. Each polarization of the lower sideband was connected to the Millimeter AutoCorrelator (MAC) spectrometer (denoted MAC 11 and MAC 12) with $0.02 \mathrm{~km} \mathrm{~s}^{-1}(6.1 \mathrm{kHz})$ resolution. The FWHM beam size at this frequency was $67 !$. 6 . Each source was observed for 60 minutes of total integration time where we position-switched between our source and an off position every $30 \mathrm{~s}$. We identified clean off positions (no $\mathrm{HCO}^{+} J=1-0$ emission at the velocities of our sources) along the Galactic plane every half-degree. Observations of Jupiter and Venus were used to calibrate the spectra from the $T_{A}^{*}$ scale to $T_{\mathrm{mb}}$ scale during each shift (Mangum 1993). Figure 2 shows the beam efficiencies for each polarization. The median $\eta_{\mathrm{mb}}=0.787$ for MAC 11 and $\eta_{\mathrm{mb}}=0.801$ for MAC 12. Our average baseline rms was $\sigma_{T_{\mathrm{mb}}}=0.067 \mathrm{~K}$ for the complete survey.

Sources were selected blindly from the starless clump candidate list in Svoboda et al. (2016) that had $\mathrm{NH}_{3}(1,1)$ detections. The average distance of the sources was $4.3 \mathrm{kpc}$, with a range from 1.2 to $11.8 \mathrm{kpc}$. Our main-beam size on the $12 \mathrm{~m}$ is approximately $1.39 \mathrm{pc}$ at that average distance. The average diameter of the sources is $2 \mathrm{pc}$, indicating that our single pointing observations cover most of the clump. Many of the sources had also been previously detected in $\mathrm{HCO}^{+} 3-2$

\footnotetext{
2 This project was the group radio observing project of the undergraduate Astronomy Club at the University of Arizona.
} 
Table 1

Starless Clump Candidates

\begin{tabular}{|c|c|c|c|c|c|c|c|}
\hline Source & $\begin{array}{c}\alpha \\
(\mathrm{hh}: \mathrm{mm}: \mathrm{ss})^{\mathrm{a}}\end{array}$ & $\begin{array}{c}\delta \\
\text { (dd:mm:ss) }\end{array}$ & $\begin{array}{c}\text { Distance } \\
(\mathrm{kpc})^{\mathrm{b}}\end{array}$ & $\begin{array}{c}v_{\mathrm{LSR}}\left(\mathrm{HCO}^{+}\right)_{\mathrm{pk}} \\
\left(\mathrm{km} \mathrm{s}^{-1}\right)\end{array}$ & $\begin{array}{c}\sigma_{v_{\text {LSR }}} \\
\left(\mathrm{km} \mathrm{s}^{-1}\right)\end{array}$ & $\delta v$ & $\sigma_{\delta v}$ \\
\hline BGPS 2427 & $18: 9: 33.88$ & $-20: 47: 0.76$ & 4.670 & 30.491 & 0.045 & -0.108 & 0.032 \\
\hline BGPS 2430 & $18: 8: 49.41$ & $-20: 40: 23.82$ & 5.013 & 22.262 & 0.094 & 0.353 & 0.035 \\
\hline BGPS 2432 & $18: 9: 44.59$ & $-20: 47: 10.21$ & 4.369 & 29.002 & 0.036 & -1.236 & 0.036 \\
\hline BGPS 2437 & $18: 10: 19.41$ & $-20: 50: 27.45$ & 4.437 & -1.464 & 0.01 & 0.305 & 0.018 \\
\hline BGPS 2533 & $18: 10: 30.29$ & $-20: 14: 44.2$ & 4.975 & 30.623 & 0.121 & -1.247 & 0.129 \\
\hline BGPS 2564 & $18: 10: 6.08$ & $-18: 46: 5.64$ & 3.013 & 29.404 & 0.038 & -0.263 & 0.069 \\
\hline BGPS 2693 & 18:11:13.56 & $-17: 44: 54.85$ & 2.103 & 18.695 & 0.115 & -0.874 & 0.230 \\
\hline BGPS 2710 & $18: 13: 49.04$ & $-17: 59: 33.25$ & 1.200 & 34.246 & 0.027 & -0.354 & 0.029 \\
\hline BGPS 2724 & $18: 14: 13.61$ & $-17: 59: 52.02$ & 1.185 & 34.428 & 0.115 & -0.543 & 0.045 \\
\hline BGPS 2732 & $18: 14: 26.85$ & $-17: 58: 50.93$ & 1.191 & 35.082 & 0.07 & -1.189 & 0.084 \\
\hline BGPS 2742 & 18:14:29.1 & $-17: 57: 21.83$ & 1.183 & 34.528 & 0.057 & -0.542 & 0.038 \\
\hline BGPS 2762 & $18: 11: 39.52$ & $-17: 32: 9.4$ & 3.304 & 18.597 & 0.062 & 0.459 & 0.046 \\
\hline BGPS 2931 & $18: 17: 27.51$ & $-17: 6: 8.42$ & 3.285 & 23.119 & 0.04 & 0.564 & 0.067 \\
\hline BGPS 2940 & $18: 17: 17.15$ & $-17: 1: 7.47$ & 3.366 & 19.903 & 0.013 & -0.061 & 0.009 \\
\hline BGPS 2945 & $18: 17: 27.35$ & $-17: 0: 23.66$ & 1.178 & 23.031 & 0.013 & 0.585 & 0.027 \\
\hline BGPS 2949 & $18: 17: 33.74$ & $-16: 59: 34.94$ & 1.279 & 22.352 & 0.025 & -0.217 & 0.040 \\
\hline BGPS 2970 & $18: 17: 5.08$ & $-16: 43: 28.66$ & 3.568 & 40.159 & 0.02 & 0.102 & 0.016 \\
\hline BGPS 2971 & $18: 16: 48.12$ & $-16: 41: 8.91$ & 1.815 & 40.371 & 0.059 & 3.434 & 0.160 \\
\hline BGPS 2976 & $18: 17: 7.84$ & $-16: 41: 14.59$ & 1.830 & 40.413 & 0.022 & 0.902 & 0.032 \\
\hline BGPS 2984 & $18: 18: 18.23$ & $-16: 44: 52.26$ & 1.855 & 17.818 & 0.026 & -1.170 & 0.062 \\
\hline BGPS 2986 & $18: 18: 29.68$ & $-16: 44: 50.69$ & 2.014 & 20.446 & 0.017 & 0.660 & 0.025 \\
\hline BGPS 3018 & $18: 19: 13.88$ & $-16: 35: 16.47$ & 6.522 & 19.27 & 0.045 & 0.281 & 0.043 \\
\hline BGPS 3030 & $18: 19: 19.68$ & $-16: 31: 39.82$ & 1.784 & 18.887 & 0.027 & -0.180 & 0.046 \\
\hline BGPS 3110 & $18: 20: 16.27$ & $-16: 8: 51.13$ & 2.005 & 17.217 & 0.006 & -0.219 & 0.008 \\
\hline BGPS 3114 & $18: 20: 31.5$ & $-16: 8: 37.8$ & 1.848 & 23.525 & 0.016 & 0.229 & 0.005 \\
\hline BGPS 3117 & $18: 20: 6.68$ & $-16: 4: 45.75$ & 2.001 & 18.519 & 0.022 & -0.032 & 0.028 \\
\hline BGPS 3118 & $18: 20: 16.17$ & $-16: 5: 50.72$ & 2.001 & 16.214 & 0.017 & -0.557 & 0.030 \\
\hline BGPS 3125 & $18: 20: 6.11$ & $-16: 1: 58.02$ & 3.466 & 18.139 & 0.046 & -4.215 & 0.216 \\
\hline BGPS 3128 & $18: 20: 35.27$ & $-16: 4: 53.81$ & 4.170 & 19.044 & 0.011 & -0.396 & 0.029 \\
\hline BGPS 3129 & $18: 20: 12.99$ & $-16: 0: 24.13$ & 4.289 & 18.812 & 0.046 & -0.781 & 0.080 \\
\hline BGPS 3134 & $18: 19: 52.72$ & $-15: 56: 1.56$ & 4.083 & 20.658 & 0.004 & 0.139 & 0.010 \\
\hline BGPS 3139 & $18: 20: 34.24$ & $-15: 58: 14$ & 5.408 & 21.103 & 0.037 & -0.805 & 0.057 \\
\hline BGPS 3151 & $18: 20: 23.19$ & $-15: 39: 31.96$ & 3.379 & 38.86 & 0.041 & -0.614 & 0.048 \\
\hline BGPS 3220 & $18: 24: 57.03$ & $-13: 20: 32.39$ & 3.874 & 46.916 & 0.024 & 0.190 & 0.009 \\
\hline BGPS 3243 & $18: 25: 32.74$ & $-13: 1: 31.05$ & 4.597 & 68.069 & 0.019 & -0.547 & 0.030 \\
\hline BGPS 3247 & $18: 25: 14.45$ & $-12: 54: 16.74$ & 4.447 & 44.886 & 0.2 & -0.344 & 0.244 \\
\hline BGPS 3276 & $18: 26: 24.92$ & $-12: 49: 30.07$ & 3.379 & 66.515 & 0.047 & -0.521 & 0.046 \\
\hline BGPS 3300 & $18: 26: 28.42$ & $-12: 37: 3.98$ & 11.668 & 62.427 & 0.031 & -0.704 & 0.031 \\
\hline BGPS 3302 & $18: 27: 15.23$ & $-12: 42: 56.45$ & 11.785 & 64.66 & 0.044 & -0.967 & 0.033 \\
\hline BGPS 3306 & $18: 23: 34.02$ & $-12: 13: 52.79$ & 4.777 & 57.082 & 0.016 & -0.030 & 0.023 \\
\hline BGPS 3312 & $18: 25: 44.52$ & $-12: 28: 34.11$ & 5.271 & 47.098 & 0.012 & -0.292 & 0.100 \\
\hline BGPS 3315 & $18: 25: 33.24$ & $-12: 26: 50.63$ & 4.793 & 47.678 & 0.021 & 2.111 & 0.184 \\
\hline BGPS 3344 & $18: 26: 40$ & $-12: 25: 15.81$ & 4.314 & 65.583 & 0.19 & -0.132 & 0.227 \\
\hline BGPS 3442 & $18: 28: 13.51$ & $-11: 40: 44.94$ & 3.442 & 67.007 & 0.003 & 1.165 & 0.022 \\
\hline BGPS 3444 & $18: 28: 27.26$ & $-11: 41: 33.99$ & 3.297 & 69.749 & 0.082 & 0.061 & 0.081 \\
\hline BGPS 3475 & $18: 28: 28.28$ & $-11: 6: 44.16$ & 3.426 & 77.643 & 0.27 & 0.641 & 0.108 \\
\hline BGPS 3484 & $18: 29: 15.74$ & $-10: 58: 28.73$ & 3.484 & 56.316 & 0.023 & -0.017 & 0.041 \\
\hline BGPS 3487 & $18: 29: 22.77$ & $-10: 58: 1.69$ & 3.490 & 53.283 & 0.061 & -0.459 & 0.073 \\
\hline BGPS 3534 & $18: 30: 33.45$ & $-10: 24: 19$ & 3.225 & $\ldots$ & $\ldots$ & $\ldots$ & $\ldots$ \\
\hline BGPS 3604 & $18: 30: 43.92$ & $-9: 34: 42.15$ & 11.010 & 50.725 & 0.013 & -0.770 & 0.017 \\
\hline BGPS 3606 & $18: 29: 41.95$ & $-9: 24: 49.1$ & 4.214 & 49.143 & 0.028 & -0.419 & 0.054 \\
\hline BGPS 3608 & $18: 31: 54.82$ & $-9: 39: 5.03$ & 4.081 & 61.869 & 0.121 & -0.597 & 0.041 \\
\hline BGPS 3627 & $18: 31: 42.32$ & $-9: 24: 29.17$ & 4.169 & 82.71 & 0.126 & 1.181 & 0.115 \\
\hline BGPS 3656 & $18: 32: 49.54$ & $-9: 21: 29.26$ & 3.906 & 77.655 & 0.061 & 0.215 & 0.042 \\
\hline BGPS 3686 & $18: 34: 14.58$ & $-9: 18: 35.84$ & 2.939 & 76.927 & 0.121 & -0.301 & 0.103 \\
\hline BGPS 3705 & $18: 34: 32.69$ & $-9: 14: 9.4$ & 3.116 & 63.207 & 0.013 & 0.771 & 0.048 \\
\hline BGPS 3710 & $18: 34: 20.55$ & $-9: 10: 1.94$ & 2.505 & 74.405 & 0.024 & -0.113 & 0.013 \\
\hline BGPS 3716 & $18: 34: 24.15$ & $-9: 8: 3.6$ & 3.146 & 75.039 & 0.082 & -0.225 & 0.027 \\
\hline BGPS 3736 & $18: 33: 28.22$ & $-8: 55: 4.36$ & 5.031 & 65.62 & 0.016 & 0.139 & 0.018 \\
\hline BGPS 3822 & $18: 33: 32.06$ & $-8: 32: 26.27$ & 3.370 & 55.14 & 0.045 & 0.389 & 0.035 \\
\hline BGPS 3833 & $18: 33: 36.5$ & $-8: 30: 50.7$ & 4.493 & 56.113 & 0.044 & 0.403 & 0.036 \\
\hline BGPS 3892 & $18: 35: 59.74$ & $-8: 38: 56.48$ & 5.300 & 62.643 & 0.027 & -0.816 & 0.015 \\
\hline BGPS 3922 & $18: 33: 40.98$ & $-8: 14: 55.3$ & 9.876 & 89.299 & 0.021 & 0.071 & 0.022 \\
\hline
\end{tabular}


Table 1

(Continued)

\begin{tabular}{|c|c|c|c|c|c|c|c|}
\hline Source & $\begin{array}{c}\alpha \\
(\mathrm{hh}: \mathrm{mm}: \mathrm{ss})^{\mathrm{a}}\end{array}$ & $\begin{array}{c}\delta \\
(\mathrm{dd}: \mathrm{mm}: \mathrm{ss})\end{array}$ & $\begin{array}{l}\text { Distance } \\
(\mathrm{kpc})^{\mathrm{b}}\end{array}$ & $\begin{array}{c}v_{\mathrm{LSR}}\left(\mathrm{HCO}^{+}\right)_{\mathrm{pk}} \\
\left(\mathrm{km} \mathrm{s}^{-1}\right)\end{array}$ & $\begin{array}{c}\sigma_{v \text { LSR }} \\
\left(\mathrm{km} \mathrm{s}^{-1}\right)\end{array}$ & $\delta v$ & $\sigma_{\delta v}$ \\
\hline BGPS 3924 & 18:34:51.17 & $-8: 23: 40.02$ & 5.782 & 81.11 & 0.029 & -0.089 & 0.017 \\
\hline BGPS 3982 & $18: 34: 30.79$ & $-8: 2: 7.36$ & 11.582 & 54.019 & 0.054 & 0.109 & 0.073 \\
\hline BGPS 4082 & $18: 35: 10.07$ & $-7: 39: 43.55$ & 5.084 & 98.994 & 0.027 & -0.433 & 0.024 \\
\hline BGPS 4085 & 18:33:57.05 & $-7: 29: 31.43$ & 5.087 & 96.409 & 0.025 & -0.131 & 0.026 \\
\hline BGPS 4095 & $18: 35: 4$ & $-7: 36: 6.46$ & 5.353 & 114.384 & 0.06 & 1.359 & 0.061 \\
\hline BGPS 4140 & $18: 36: 49.66$ & $-7: 40: 36.83$ & 3.617 & 96.106 & 0.025 & 0.114 & 0.032 \\
\hline BGPS 4145 & $18: 36: 52.95$ & $-7: 39: 49.2$ & 4.985 & 96.52 & 0.023 & -0.010 & 0.022 \\
\hline BGPS 4191 & $18: 37: 4.58$ & $-7: 33: 12.26$ & 5.123 & 97.211 & 0.019 & -0.226 & 0.016 \\
\hline BGPS 4230 & $18: 35: 50.85$ & $-7: 12: 23.58$ & 5.034 & 109.337 & 0.052 & 1.604 & 0.065 \\
\hline BGPS 4294 & $18: 38: 51.58$ & $-6: 55: 36.52$ & 5.688 & 58.936 & 0.146 & 2.106 & 0.115 \\
\hline BGPS 4297 & $18: 38: 56.37$ & $-6: 55: 8.44$ & 4.973 & 59.254 & 0.025 & 0.464 & 0.024 \\
\hline BGPS 4375 & 18:39:10.19 & $-6: 21: 15.9$ & 3.793 & 92.913 & 0.014 & -0.160 & 0.019 \\
\hline BGPS 4396 & $18: 38: 34.74$ & $-5: 56: 43.97$ & 4.266 & 112.998 & 0.027 & 0.241 & 0.026 \\
\hline BGPS 4402 & $18: 39: 28.64$ & $-5: 57: 58.57$ & 4.285 & 100.149 & 0.124 & 0.679 & 0.091 \\
\hline BGPS 4422 & $18: 38: 47.88$ & $-5: 36: 16.38$ & 3.917 & 111.503 & 0.049 & 0.728 & 0.054 \\
\hline BGPS 4472 & $18: 41: 17.32$ & $-5: 9: 56.83$ & 3.216 & 47.937 & 0.084 & 1.138 & 0.093 \\
\hline BGPS 4732 & $18: 44: 23.4$ & $-4: 2: 1.21$ & 3.782 & 89.609 & 0.074 & 1.020 & 0.060 \\
\hline BGPS 4827 & $18: 44: 42.45$ & $-3: 44: 21.63$ & 4.928 & 89.611 & 0.04 & 3.011 & 0.082 \\
\hline BGPS 4841 & $18: 42: 15.65$ & $-3: 22: 26.19$ & 4.266 & 83.534 & 0.031 & -0.288 & 0.022 \\
\hline BGPS 4902 & $18: 46: 11.36$ & $-3: 42: 55.73$ & 4.656 & 84.262 & 0.136 & 0.050 & 0.056 \\
\hline BGPS 4953 & $18: 45: 51.82$ & $-3: 26: 24.16$ & 5.502 & 90.192 & 0.054 & -0.328 & 0.033 \\
\hline BGPS 4962 & $18: 45: 59.61$ & $-3: 25: 14.53$ & 6.092 & 88.765 & 0.078 & 0.493 & 0.091 \\
\hline BGPS 4967 & $18: 43: 27.8$ & $-3: 5: 14.94$ & 3.681 & 81.2 & 0.021 & 0.697 & 0.025 \\
\hline BGPS 5021 & $18: 44: 37.07$ & $-2: 55: 4.4$ & 5.181 & 78.77 & 0.072 & -0.843 & 0.049 \\
\hline
\end{tabular}

Notes.

${ }^{\text {a }}$ All coordinates are epoch $\mathrm{J} 2000.0$.

${ }^{\mathrm{b}}$ Distances are the maximum likelihood distance of the distance probability density function (DPDF) in Svoboda et al. (2016).

(Shirley et al. 2013), but since that survey sacrificed spectral resolution (only $1.1 \mathrm{~km} \mathrm{~s}^{-1}$ channel width) for wide bandwidth, we are unable to use the existing 3-2 observations to systematically search for inflow in the SCC sample. Throughout this paper, we use the BGPS v2.0.1 catalog number (Ginsburg et al. 2013) for the source name (i.e., BGPS 4029). The observed sample is 101 objects in the first quadrant of the Galactic plane, with a median distance of $4.1 \mathrm{kpc}$ (see Table 1). Figure 3 shows the comparison of the mass and peak mass surface density between the $12 \mathrm{~m}$ sample and the complete sample of SCCs with well-constrained distances (see Ellsworth-Bowers et al. 2015, for an explanation of how distances are determined toward BGPS clumps). The $12 \mathrm{~m}$ sample has a median mass of $300 M_{\odot}$ and is representative of the full range of observed mass, spanning masses up to $5550 M_{\odot}$ (BGPS 3114); however, the observed $12 \mathrm{~m}$ sample is biased to higher peak mass surface densities by a factor of $\sim 2$ compared to the complete SCC sample with well-constrained distances (see Svoboda et al. 2016).

For the best inflow candidates (Section 3), we also made observations in $\mathrm{H}^{13} \mathrm{CO}^{+} 1-0$ at $86,754.2884 \mathrm{MHz}$. The optically thinner $\mathrm{H}^{13} \mathrm{CO}^{+}$observations were directly compared to the $\mathrm{v}_{\mathrm{LSR}}$ from $\mathrm{NH}_{3}$ observations (Svoboda et al. 2016) as a secondary check that an optically thin line peaks in the $\mathrm{HCO}^{+} 1-0$ absorption dip. The spectrometer setup and subsequent analysis are identical to that of $\mathrm{HCO}^{+} 1-0$ observations.

\section{Results}

\subsection{Line Asymmetry Statistics}

We observed a total of 101 starless clump candidates in $\mathrm{HCO}^{+} J=1-0$. Two of the spectra were not used for the subsequent analysis because they were nondetections at the $\mathrm{NH}_{3}$ velocity (BGPS 3534 and BGPS 5183). All spectra were 


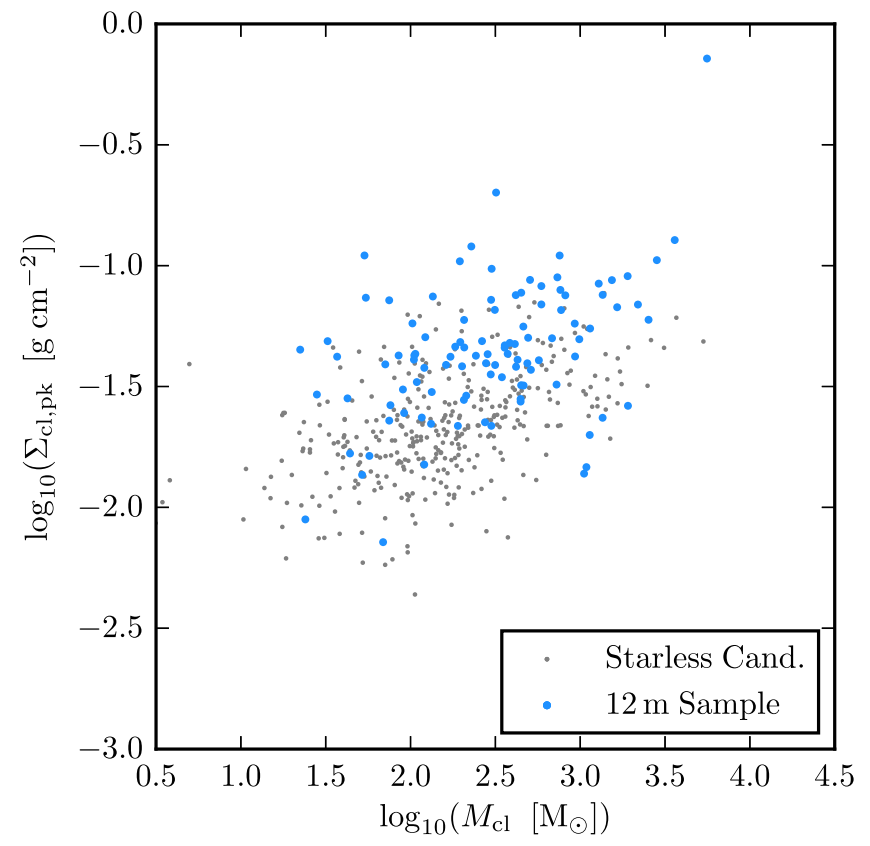

Figure 3. Mass and peak mass surface density of BGPS starless clump candidates with well-constrained distances. Each point corresponds to the median value of the mass and peak mass surface density probability density functions (see Svoboda et al. 2016). "12 m Sample" is the subset of sources observed in this paper.

visually analyzed to search for blue asymmetric line profiles with self-absorption dips (Figures 4 and 5). If $\mathrm{NH}_{3}$ peaked in the self-absorption dip of a blue asymmetric profile, it was considered a candidate for inflow. We found that six of the 101 sources $(6 \%)$ were good infall candidates. Their line profiles are shown in Figure 6. There are some negative velocities, which would suggest emission in the off positions, but any negative peaks are located far from the source spectra and will not affect the analysis.

We checked these candidates by also observing the optically thinner tracer $\mathrm{H}^{13} \mathrm{CO}^{+} 1-0$. In all cases except for BGPS 2432, the $\mathrm{H}^{13} \mathrm{CO}^{+}$peak velocity agreed with the $\mathrm{NH}_{3}$ velocity within $0.2 \mathrm{~km} \mathrm{~s}^{-1}$. Even in the case of BGPS 2432, where the velocity difference between $\mathrm{H}^{13} \mathrm{CO}^{+}$and $\mathrm{NH}_{3}$ was $+0.57 \mathrm{~km} \mathrm{~s}^{-1}$, both lines still peak within the $\mathrm{HCO}^{+}$absorption dip. Thus, $\mathrm{H}^{13} \mathrm{CO}^{+}$ observations confirm that an optically thinner line peaks in the absorption dip for all six candidates. This result also indicates that the $\mathrm{NH}_{3}(1,1)$ and $(2,2)$ transitions are good tracers of the systemic velocity of the clumps, partly due to their multiple hyperfine lines with different optical depths. In the subsequent analysis, we consider the six sources (BGPS 2432, BGPS 3300, BGPS 3302, BGPS 3604, BGPS 4029, and BGPS 5021) as inflow candidates.

All of the six inflow candidates have an $\mathrm{HCO}^{+}$absorption dip that is slightly red of the $\mathrm{NH}_{3}$ or $\mathrm{H}^{13} \mathrm{CO}^{+}$peak velocities. This effect has been observed in other inflow surveys (He et al. 2016; Jin et al. 2016). As described in Jin et al. (2016), global collapse (i.e., in competitive accretion models) could potentially create a redshifted absorption dip. This can be tested with higher-resolution observations; at the single-dish resolution of observations in this paper, these sources remain only candidates for global inflow.

A visual analysis of the spectra is not a robust quantitative measure of the asymmetry in the line profile; therefore, we use $\delta v$ to measure the velocity shift between $\mathrm{HCO}^{+}$and $\mathrm{NH}_{3} . \delta v$ was first defined by Mardones et al. (1997):

$$
\delta v=\frac{v_{\mathrm{HCO}^{+}}-v_{\mathrm{NH}_{3}}}{\Delta v_{\mathrm{NH}_{3}}}
$$

where $v_{\mathrm{HCO}^{+}}$is the peak $\mathrm{HCO}^{+}$velocity, $v_{\mathrm{NH}_{3}}$ is the peak $\mathrm{NH}_{3}$ velocity, and $\Delta v_{\mathrm{NH}_{3}}$ is the FWHM line width of the $\mathrm{NH}_{3}$ lines. Ammonia parameters were taken from Svoboda et al. (2016). With this measure, if $\delta v$ is negative, it has blue asymmetry. If it is positive, it has a red asymmetry.

Figure 7 shows the histogram of $\delta v$ values for the observed SCC sample (also see Table 1). Most of the sources are clustered within the range of $-2 \leqslant \delta v \leqslant 2$. One notable exception is the source BGPS 3125, with a $\delta v<-4$. Visual inspection of the source indicates that the $\mathrm{HCO}^{+} 1-0$ is a rare example of a triplepeaked spectrum with the $\mathrm{NH}_{3}$ well aligned with a weaker peak in $\mathrm{HCO}^{+}$. Therefore, BGPS 3125 is not a good infall candidate, and its large negative $\delta v$ should be ignored. All of the six best infall candidates have $\delta v<-0.48$. Of the remaining sources with $\delta v<-0.5$, eight of the sources are single peaked in $\mathrm{HCO}^{+}$ with no self-absorption dip, two sources are double peaked but the peaks are nearby equal in height (BGPS 2533 and BGPS 3151 ), one source is double peaked but a poor inflow candidate owing to $\mathrm{NH}_{3}$ not peaking within the self-absorption dip (BGPS 2984), and three sources have more than two peaks in $\mathrm{HCO}^{+}$. See Figures 4 and 5 for spectra of all observed clumps.

There is a very slight bias of blue asymmetry over red asymmetry in our observed $\delta v$ distribution. We use the definition of blue excess given in Mardones et al. (1997):

$$
E=\frac{N_{\text {blue }}-N_{\text {red }}}{N_{\text {total }}},
$$

where $N_{\text {blue }}$ is the number of blue asymmetric profiles, $N_{\text {red }}$ is red asymmetric, and $N_{\text {total }}$ is the total number of clumps in our sample. Using the limit that a $\delta v$ of less than -0.25 is a significant blue asymmetry and one of greater than 0.25 is a significant red asymmetry (since the \pm 0.25 threshold is approximately 5 times our median error in $\delta v$ ), we then have $N_{\text {blue }}=35$ clumps and $N_{\text {red }}=32$ clumps. This leaves another 32 clumps that had a $\delta v$ value between -0.25 and 0.25 (the total number of clumps with a valid $\delta v$ value is 99 clumps). Using these values, we come to a blue excess of $E=0.03$.

Compared to other surveys that calculated the blue excess also using $\mathrm{HCO}^{+}$, our value for blue excess is on the low end. Fuller et al. (2005), Purcell et al. (2006), Wu et al. (2007), He et al. (2015), and Rygl et al. (2013) have each completed a survey of various stages of star formation while using the chemical tracer $\mathrm{HCO}^{+}$. Four out of the five of the surveys found excess values near $E=0.2$, while one found a value near ours at $E=0.02$. He et al. (2015) and He et al. (2016) used the MALT90 survey data (Jackson et al. 2013) to calculate their blue excess, and their prestellar objects were most similar to our sample of SCCs; clumps with no mid-infrared sources were identified by a flux cut in the ATLASGAL survey. We found a larger fraction of objects with red excess as compared to their results. We should note that there is significant difference in the definition of an inflow candidate given by He et al. (2016) and our paper; namely, we require the line profile to have a blue asymmetry with a self-absorption profile, while the He et al. (2016) paper only required that the source have $\delta v<-0.25$ in at least one optically thick tracer and no red skewed profiles in any optically thick tracer. We find 

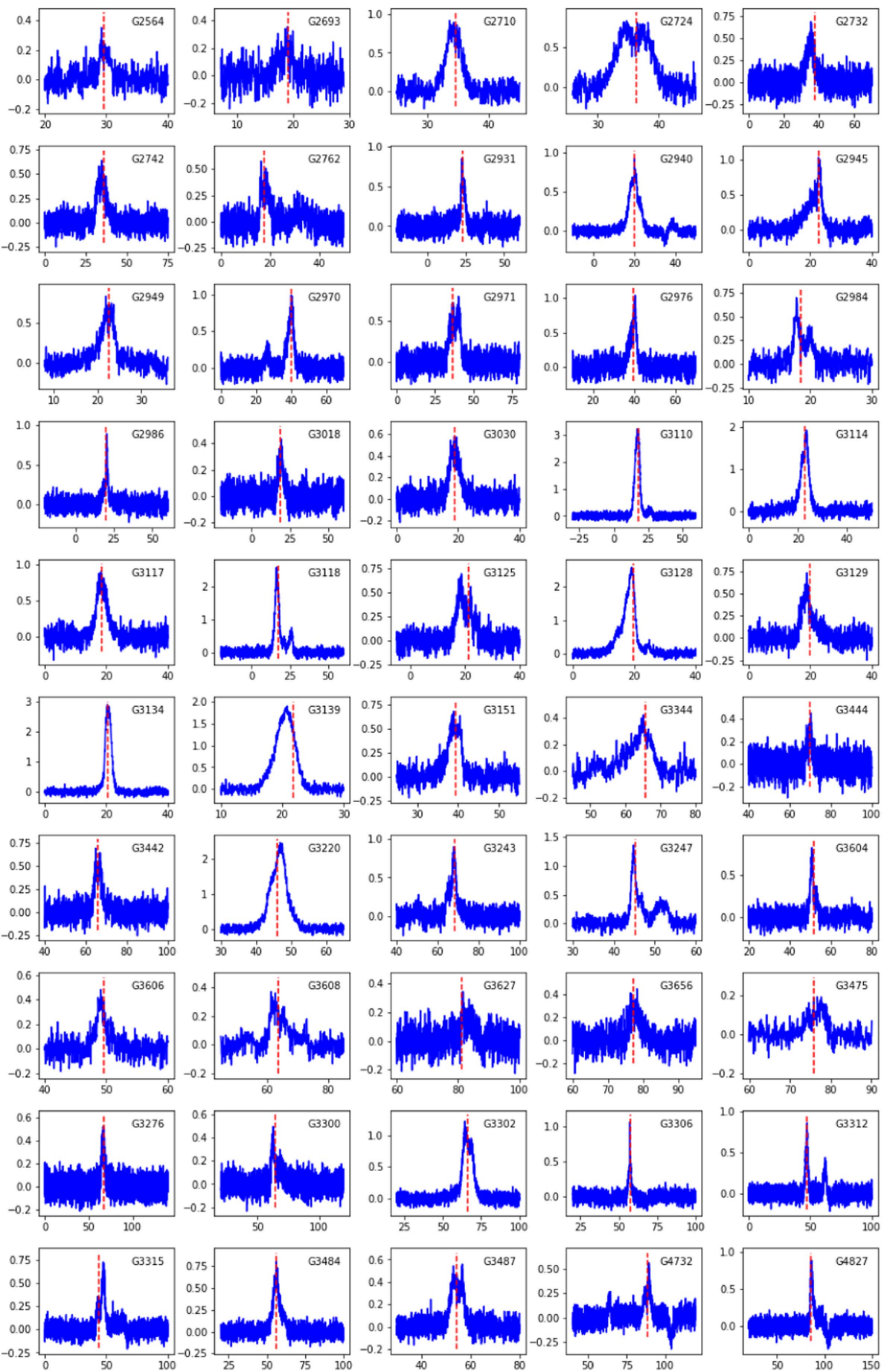

Figure 4. Line profiles of the first half of the 99 clumps that have detections in $\mathrm{HCO}^{+}$. The $y$-axis is in $\mathrm{km} \mathrm{s}^{-1}$, and the $x$-axis is in kelvin. The red dashed line shows the observed ammonia peak.

several sources with $\delta v<-0.5$ but that are single peaked in $\mathrm{HCO}^{+} 1-0$. With a single pointing survey and a large beam that encompasses the entire clump, we cannot confidently claim that these large negative $\delta v$ single-peaked HCO $1-0$ sources are good inflow candidates; the observed velocity offset between $\mathrm{HCO}^{+}$and $\mathrm{NH}_{3}$ may be due to variations in the chemical or excitation structure of the clumps. In addition to this, our sample was selected blindly from SCCs that had previously been detected in $\mathrm{NH}_{3}$ emission, while the MALT90 prestellar clumps were selected from a flux cut and therefore represent the brightest ATLASGAL clumps in that category.
The only study with a blue excess similar to ours is that of Purcell et al. (2006), which observed methanol masers associated with massive YSOs. It is likely that their $E=0.02$ is contaminated by strong outflow motions associated with massive protostars that can create a red asymmetric line profile. Our survey is unique in that we are observing the earliest stage of star formation where there should not be strong contamination due to outflows from intermediate- or high-mass stars. With our value of $E=0.03$ we see that the earliest stages of star formation do not strongly favor blue or red asymmetric $\mathrm{HCO}^{+} 1-0$ line profiles for BGPS starless clump candidates. 

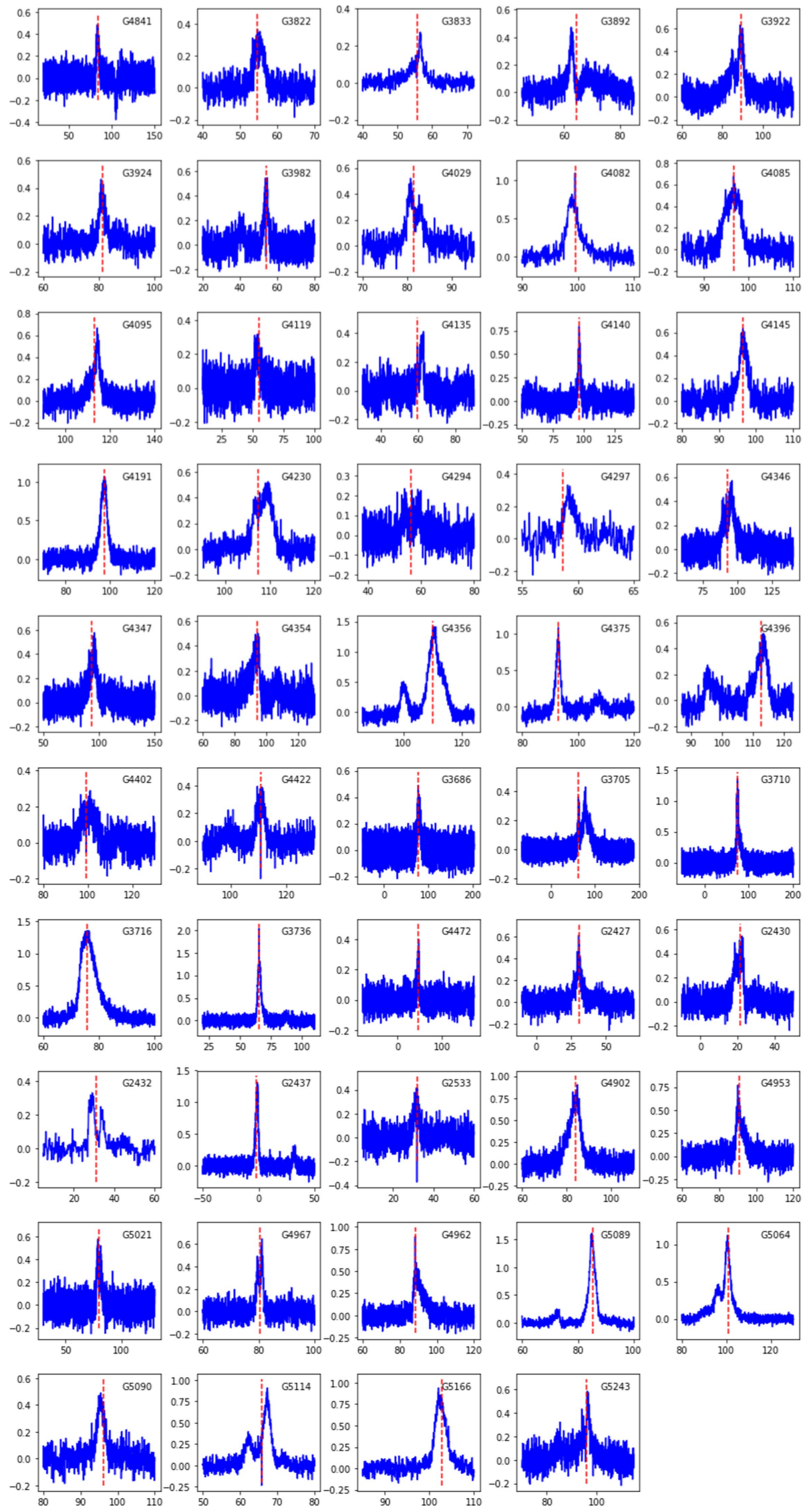

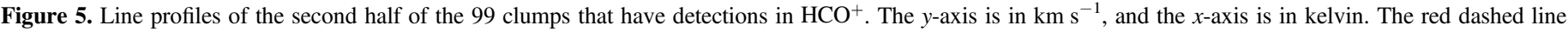
shows the observed ammonia peak. 

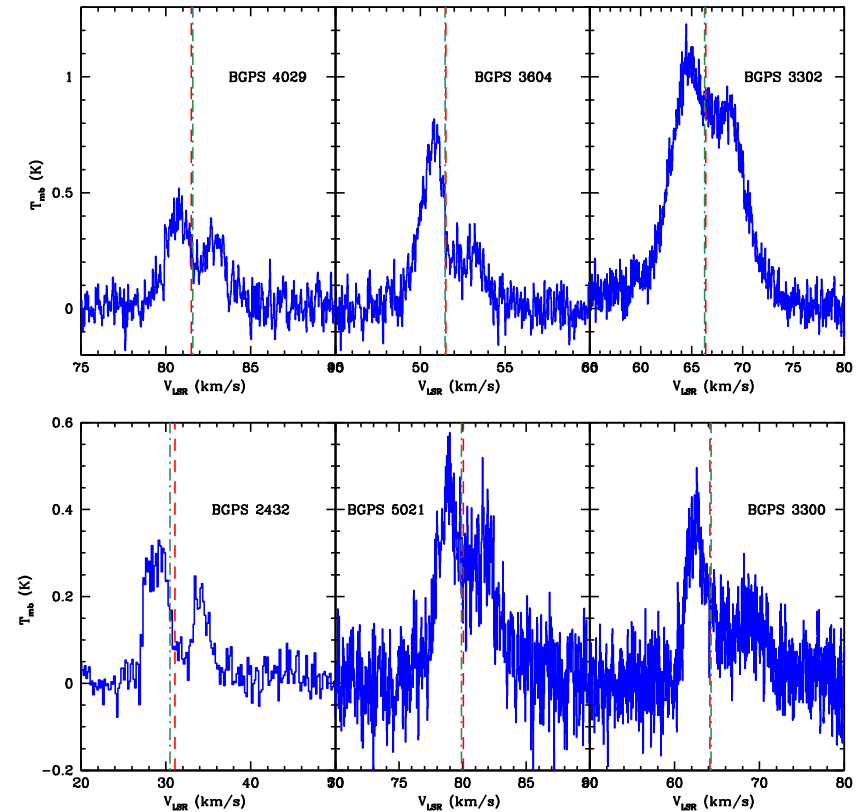

Figure 6. Line profiles of our best inflow candidates. Each profile has blue asymmetry with an $\mathrm{NH}_{3}$ peak (shown as the red dashed line) and an $\mathrm{H}^{13} \mathrm{CO}^{+}$ peak (shown as a green dotted-dashed line) in the self-absorption dip. These were the only sources that met these two criteria and had reasonable inflow velocities as modeled in Section 4.

\subsection{Comparison with Physical Properties of the Clumps}

We compared $\delta v$ to the virial parameter and clump mass as seen in Figure 5. There is no strong relationship between $\delta v$ and the mass, meaning that given a certain clump's mass we cannot accurately predict that same clump's $\delta v$ value. We might have expected there to be a slight relationship that suggested that more massive clumps were more likely to have a blue $\delta v$ value, since the more massive clumps might be more likely to be gravitationally collapsing, but we observe no trend.

Another indicator of the potential for gravitational collapse is the virial parameter, $\alpha_{\text {vir }}$. An $\alpha_{\text {vir }}<\sim 2$ signifies that a clump is gravitationally bound and may undergo collapse. Many of our clumps are defined as gravitationally bound, but the $\delta v$ value once again does not correlate with this parameter. It is important to note that in our calculation of $\alpha_{\text {vir }}$ we only account for kinetic and potential energy and not for external pressure or magnetic field support. As discussed in Svoboda et al. (2016), a modest magnetic field of only a few hundred microgauss is needed to raise the typical SCC virial parameter above 2. A proper virial analysis also needs to consider the weight of the surrounding cloud on the clump and turbulent surface pressure (see Kirk et al. 2017).

We were unable to find any significant trend between $\delta v$ and physical parameters of the clumps. This statement includes the six best inflow candidates, which are not strongly clustered in mass or virial parameter. Our lack of correlation is in contrast to the recent survey of 17 massive clumps that are $70 \mu \mathrm{m}$ dark by Traficante et al. (2018), which did find a correlation between $\mathrm{HCO}^{+} 1-0$ line asymmetry (defined in a different method than used here) and the peak mass surface density of the clumps; however, the peak surface densities in our survey are generally lower (see Figure 3) than the $0.1 \mathrm{~g} \mathrm{~cm}^{-3}$ threshold for which they observe more asymmetric line profiles.

\section{Analysis and Discussion}

Only $6 \%$ of observed starless clump candidates show a signature of inflow in $\mathrm{HCO}^{+} 1-0$. This result, taken without caveats, indicates either that large-scale inflow motions are a rare occurrence in our Galaxy or that a global initial inflow process during the starless phase is very quick and is therefore only observable in a few percent of clumps. Svoboda et al. (2016) estimated the starless phase lifetime of clumps and showed that the phase lifetime varied inversely with the mass of the clumps. If the low percentage of observable inflow signatures is attributable to a brief massive inflow phase, then the Svoboda et al. (2016) relationship indicates that this massive global inflow starless phase lasts $\sim 60,000 \mathrm{yr}$ for the median inflow candidate in our sample. However, we must consider the caveats, as there are many different factors that could result in nondetection of inflow. The detection of a blue asymmetric profile requires a specific set of conditions, namely, increasing excitation temperature along the line of sight, being optically thick along the line of sight, and a favorable line-ofsight velocity field. Simulations of global collapse show that there is a directional efficiency factor of up to $\sim 50 \%$ for observing blue asymmetric line profiles with common dense gas tracers (Smith et al. 2012, 2013), meaning that our observed inflow detection rate of $6 \%$ could be $12 \%$ in reality. Furthermore, we are only sensitive to motions that are fast enough to produce a measurable blue asymmetry, meaning that a slow flow comparable to the FWHM line width observed in our large beam will not be detected. Radiative transfer simulations (Smith et al. 2013) also show that larger beam sizes suppress blue asymmetries. Thus, our observational result is likely a lower limit to the number of clumps with detectable inflow. In this section, we shall model and analyze the profiles of the best inflow candidates, calculate the mass inflow rate, and calculate the total change in mass of the clump in a freefall time.

We model our best inflow candidates using the analytic HILL5 model from De Vries \& Myers (2005) with five parameters: $\tau_{0}$, the optical depth at the peak observed line temperature $T_{\mathrm{pk}} ; v_{\mathrm{in}}$, the inflow velocity; $v_{\mathrm{LSR}} ;$ and $\sigma$, the line width. The HILL5 model solves the equation of radiative transfer with the assumption that the excitation temperature is a linear function of optical depth (a "HILL" model). De Vries \& Myers (2005) found that the five-parameter version of the HILL model most consistently and accurately recovered the inflow velocity when fit to synthetic spectra created from radiative transfer models compared to other parameterizations of the HILL model and the simpler "two-layer" model of Myers et al. (1996). We apply Bayesian parameter estimation to fit the HILL5 model independently to each $\mathrm{HCO}^{+} 1-0$ spectrum. We use the affine-invariant Markov chain Monte Carlo (MCMC) ensemble sampler emcee (Foreman-Mackey et al. 2013) to maximize its log-likelihood function

$$
\ln p(T \mid \boldsymbol{\theta})=-\frac{1}{2} \sum_{\mathrm{n}}\left[\frac{\left(T_{\mathrm{n}}-f_{\mathrm{h} 5}(\boldsymbol{\theta})\right)^{2}}{\sigma_{T}^{2}}\right]
$$

over each channel $n$ in the spectrum with observed main-beam temperature $T_{\mathrm{n}}$, uniform baseline rms $\sigma_{T}$, and HILL5 model value (Equation (4)) for parameter set $\boldsymbol{\theta}$. Uniform priors were chosen with ranges of $\tau_{0}=0-10, \Delta v_{\mathrm{LSR}}= \pm 3 \mathrm{~km} \mathrm{~s}^{-1}, v_{\text {in }}=$ $0-1 \mathrm{~km} \mathrm{~s}^{-1}, \sigma=0-10 \mathrm{~km} \mathrm{~s}^{-1}$, and $T_{\mathrm{pk}}=0-100 \mathrm{~K}$. This range was chosen to accommodate the range of physical values while 

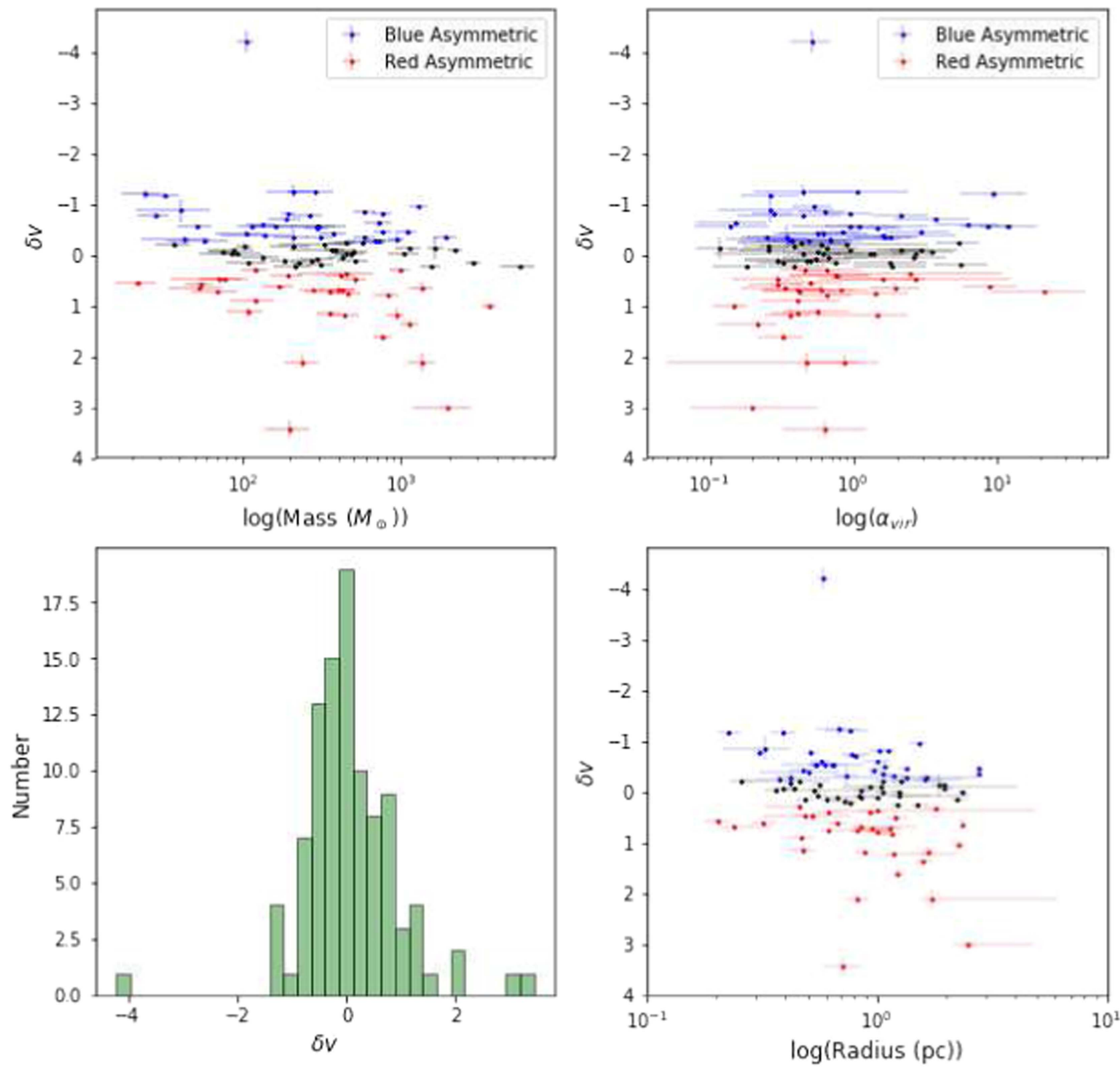

Figure 7. We compare $\delta v$ to mass (top left panel), virial parameter (top right panel), and clump radius (bottom right panel). The histogram of $\delta v$ values is shown in the bottom left panel. There is no obvious correlation present in the three $\delta v$ comparison graphs. The Spearman rank coefficient of these three plots is 0.19 for mass, -0.12 for virial parameter, and 0.18 for radius. The red points are clumps with a red asymmetry $\delta v>+0.25$, and the blue points are clumps with a blue asymmetry $\delta v<-0.25$, while black points fall in a middle range of $\delta v$.

preventing nonphysical solutions when the model spectra extend outside the data range. Initial guesses start with $\tau_{0}=1$, $\Delta v_{\mathrm{LSR}}=0 \mathrm{~km} \mathrm{~s}^{-1}, v_{\mathrm{in}}=0 \mathrm{~km} \mathrm{~s}^{-1}, \sigma=\sigma_{\mathrm{NH}_{3}} \mathrm{~km} \mathrm{~s}^{-1}$, and $T_{\mathrm{pk}}=$ $2 T_{\mathrm{HCO}^{+}} \mathrm{K}$.

We find good fits to all of the best inflow candidates (Figure 8), with inflow velocities that range from 0.25 to $0.84 \mathrm{~km} \mathrm{~s}^{-1}$. The median inflow velocity is $0.72 \mathrm{~km} \mathrm{~s}^{-1}$, and the median uncertainty from the MCMC analysis is $0.13 \mathrm{~km} \mathrm{~s}^{-1}$. These motions, if attributed to inflow, are supersonic. Unsurprisingly, the HILL5 model is consistent with zero inflow velocity in these two cases. The inflow velocities are plotted versus mass of the clumps in the Figure 9. The inflow velocities do not correlate with the mass of the clumps; however, the inflow candidates with masses below the median $300 M_{\odot}$ of the sample all tend to have the largest inflow velocities.
The inflow velocities are plotted versus mass of the clumps in Figure 7. The inflow velocities do not correlate with the mass of the clumps; however, the inflow candidates with masses below the median $300 M_{\odot}$ of the sample all tend to have the largest inflow velocities.

Using the inflow velocity estimated by our HILL5 modeling, we calculate the mass inflow rate taken from López-Sepulcre et al. (2010):

$$
\begin{aligned}
\dot{M} & =4 \pi R^{2} v_{\text {in }} \rho=\frac{3 M v_{\text {in }}}{R} \\
& =3068 \frac{M_{\odot}}{\operatorname{Myr}}\left(\frac{M}{1000 M_{\odot}}\right)\left(\frac{v_{\text {in }}}{1 \mathrm{~km} \mathrm{~s}^{-1}}\right)\left(\frac{1 p c}{R}\right) .
\end{aligned}
$$

The highest inflow rate is BGPS 3302, with $1880 M_{\odot} \mathrm{Myr}^{-1}$, and the lowest inflow rate is BGPS 4029, with $520 M_{\odot} \mathrm{Myr}^{-1}$. We 
The Astrophysical Journal, 862:63 (12pp), 2018 July 20

Callahan et al.
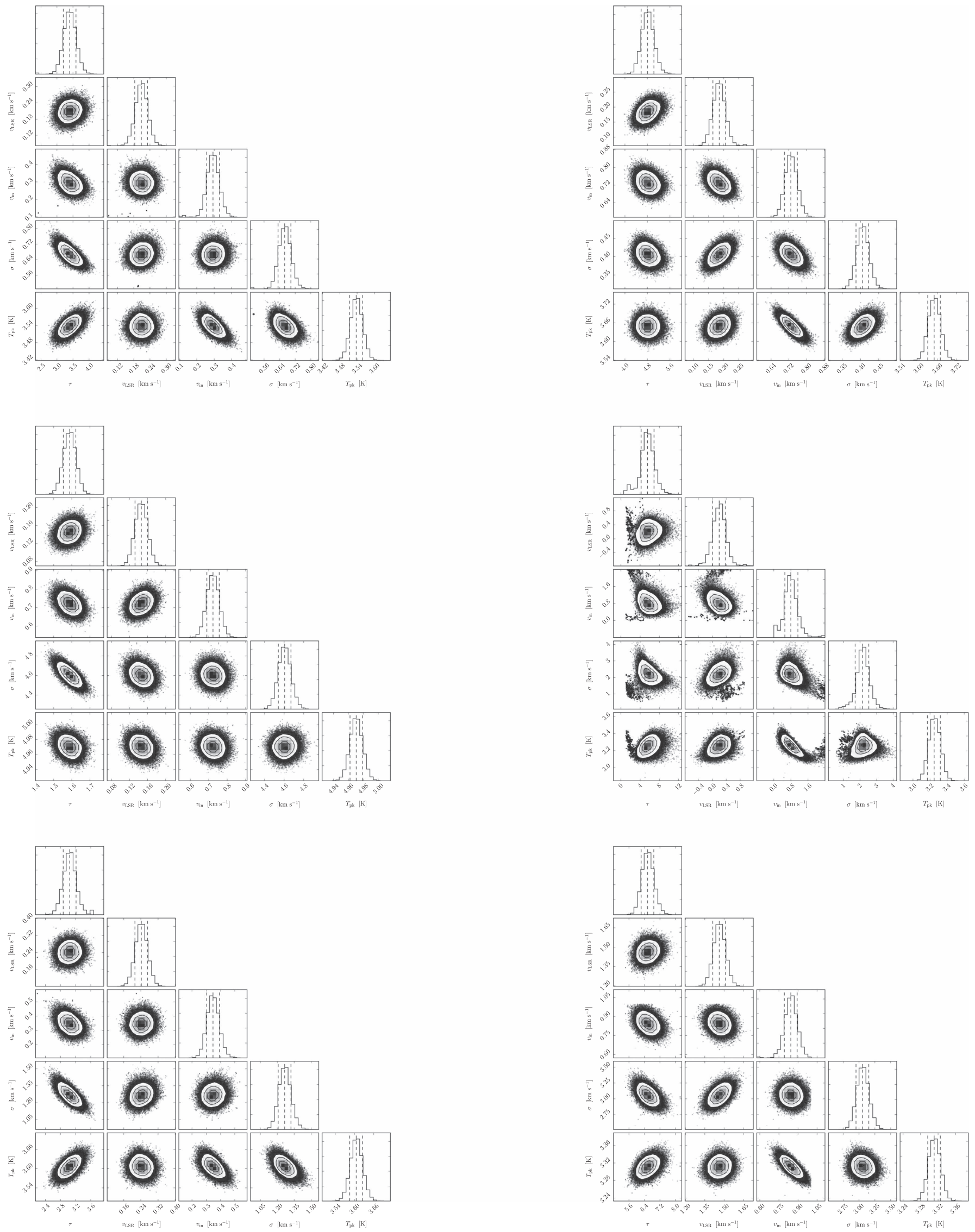

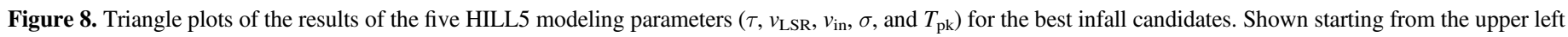
are $4029,3604,3302,2432,5021$, and 3300 ending at the lower right.

10 
Table 2

Inflow Candidates

\begin{tabular}{|c|c|c|c|c|c|c|}
\hline Source & $\begin{array}{c}v_{\text {in }} \\
\left(\mathrm{km} \mathrm{s}^{-1}\right)\end{array}$ & $\begin{array}{c}\sigma_{\text {vin }} \\
\left(\mathrm{km} \mathrm{s}^{-1}\right)\end{array}$ & $\begin{array}{c}t_{\mathrm{ff}}{ }^{\mathrm{a}} \\
(\mathrm{Myr})\end{array}$ & $\left(M_{\odot} \dot{M y r}^{-1}\right)$ & $\begin{array}{l}\Delta M \\
\left(M_{\odot}\right)\end{array}$ & $\Delta M / M$ \\
\hline BGPS 2432 & 0.718 & 0.196 & 0.661 & 823 & 544 & 2.9 \\
\hline BGPS 3300 & 0.841 & 0.165 & 0.871 & 607 & 529 & 3.2 \\
\hline BGPS 3604 & 0.728 & 0.119 & 0.704 & 748 & 526 & 2.8 \\
\hline BGPS 4029 & 0.296 & 0.130 & 0.593 & 521 & 309 & 0.7 \\
\hline BGPS 5021 & 0.347 & 0.082 & 0.694 & 567 & 393 & 1.0 \\
\hline
\end{tabular}

Note.

${ }^{\mathrm{a}}$ The median of the freefall probability density function calculated in Svoboda et al. (2016).

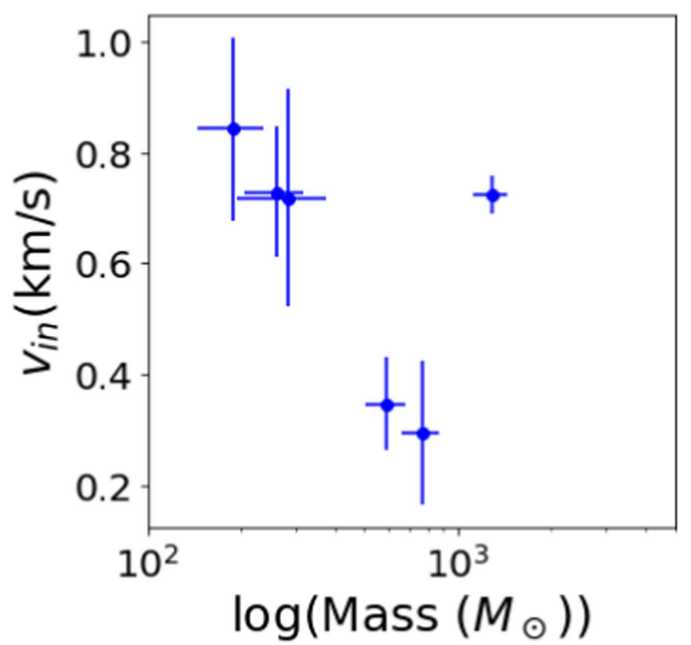

Figure 9. Here we show the relationship between infall velocity as modeled by HILL5 and clump mass. We do not find any significant correlation here. The Spearman rank correlation coefficient is -0.66 . We are working with a low number of objects, and our $\delta v$ analysis showed that there is no correlation between radius (a factor in $v_{\text {in }}$ ) and inflow likelihood as represented by $\delta v$. (See Figure 7).

are assuming complete symmetrical collapse of the clump with all of the mass of the clump participating in the inflow motions. The calculated mass inflow rate will be an overestimate if only a fraction of the mass of the clump is associated with inflow motions within the $12 \mathrm{~m}$ telescope beam. On the other hand, we only measure velocities along the line of sight, and this geometry likely underestimates the inflow velocity and subsequent mass accretion rate. It is not clear which factor would dominate, and therefore our mass accretion rates are highly uncertain.

Large-scale inflow has been observed in both high-mass and low-mass star-forming regions. Peretto et al. (2013) studied star-forming regions centered at a massive inflowing filament, similar to the type of object we are searching for. They found a mass inflow rate of $2500 \pm 1000 M_{\odot} \mathrm{Myr}^{-1}$, which overlaps with the upper range of $\dot{M}$ seen in our sample; however, this clump is known to already be forming massive stars and is not starless. Liu et al. (2017) observed large-scale inflows near $1000 M_{\odot} \mathrm{Myr}^{-1}$ from maps of $\mathrm{HCO}^{+} 1-0$ toward three protostellar BGPS clumps that were also observed in the MALT90 survey. Barnes et al. (2010) observed a dense molecular core undergoing gravitational collapse and also used Hill5 modeling and $\mathrm{HCO}^{+}$observations. They found a mass inflow rate of $3400 \pm 1700 M_{\odot} \mathrm{Myr}^{-1}$, which is a higher rate than most of our candidates but does overlap with our highest inflow rates. Kirk et al. (2013) observed a filament inflowing into a central region in the intermediate-mass Serpens starforming cloud and found that along the filament $\dot{M}=$ $30 M_{\odot} \mathrm{Myr}^{-1}$ and radially the rate was $130 M_{\odot} \mathrm{Myr}^{-1}$. These values are smaller than the range in which we observe; however, the object they were observing was lower in mass than our inflow candidates $\left(\sim 20 M_{\odot}\right.$ in the filament). It is doubtful that we could detect such low inflow rates toward BGPS SCCs given the modest line widths and our requirement that we observe a clear self-absorption dip in the $\mathrm{HCO}^{+} 1-0$ line profile. Setting a lower limit of $v_{\text {in }}=0.2 \mathrm{~km} \mathrm{~s}^{-1}$ to produce a self-absorption dip for the typical BGPS SCC line width, then for a $0.5 \mathrm{pc}, 100 M_{\odot}$ clump we have a limiting detectable mass inflow rate of $\sim 120 M_{\odot} \mathrm{Myr}^{-1}$. Thus, it is entirely possible that we have missed the types of inflow deteced in the Serpens star-forming region and are only detecting massive, large-scale flows.

The candidate inflow clumps in our survey appear to be in an earlier phase than the examples where large-scale flows are observed, which are described above. The only published inflow survey of comparable objects with calculated mass inflow rates is the study of He et al. (2016), which analyzed spectra toward prestellar clumps observed in the ATLASGAL and MALT90 surveys. The objects are classified as prestellar based on the lack of mid-infrared or far-infrared compact source emission. He et al. (2016) find a median mass inflow rate of $2600 \mathrm{M}_{\odot} \mathrm{Myr}^{-1}$, which is higher than our mass inflow estimates and comparable to the mass inflow rate observed by Peretto et al. (2013) toward a massive star-forming hub-filament complex. The He et al. (2016) inflow sample is generally more massive $\left(500 M_{\odot}\right)$ and has higher peak mass surface densities $\left(0.14 \mathrm{~g} \mathrm{~cm}^{-3}\right)$ by a factor of 3-4 than our sample. As noted in Section 3.1, our constraints to qualify a source as an inflow candidate are more stringent than in $\mathrm{He}$ et al. (2016).

For our six best inflow candidates, we calculate the change in mass of the clump assuming a constant mass accretion rate over the average clump freefall time $\left(\Delta M=\dot{M} t_{\mathrm{ff}}\right)$. Using the median freefall time for each clump calculated in Svoboda et al. (2016), we find that BGPS 4029 has the lowest $\Delta M$ of $310 M_{\odot}$ and BGPS 2432 has the highest value of $1410 M_{\odot}$. If we divide the total change in mass during the freefall time by the mass of the clump, we find that most of the clumps would double in mass over a freefall time (See Table 2). It should be noted that the freefall time corresponds to the time for the clump to collapse at the average clump volume density and corresponds to a median value of $0.7 \mathrm{Myr}$ for the inflow candidates. This timescale is longer than the median gas 
depletion timescale of $M / \dot{M}=0.5 \mathrm{Myr}$ for the inflow candidates, which may indicate that the clumps are growing in mass from the surrounding medium. While most SCCs may have a phase lifetime longer than their clump-average freefall timescale (Svoboda et al. 2016), the inflow candidates may be rare examples of clumps that are in a phase where they are embedded within a supersonic flow and are growing in mass at a significant rate.

Our single-dish survey, while a good first step for identifying promising inflow candidates, cannot exclude the possibility that observed line profiles are due to unresolved motions between dense cores within the clump. Furthermore, our analysis of the mass inflow rates has made the very simple assumption that the total mass of the clump is associated with the inflow motions. These objects are clearly interesting targets for higherresolution observations (i.e., with ALMA) that can directly address these caveats.

\section{Conclusions}

We observed 101 massive starless clump candidates from the BGPS survey using the optically thick, intermediate-density gas tracer $\mathrm{HCO}^{+} J=1-0$. We found that only six clumps $(6 \%)$ had line profiles that had a self-absorption dip at nearly the same velocity (slightly redshifted) as the peak of $\mathrm{NH}_{3}(1,1)$ emission and had an $\mathrm{HCO}^{+} 1-0$ peak intensity blueward of its self-absorption dip (blue asymmetry). The complete sample of SCCs has a small blue excess of only $E=0.03$, indicating that blue asymmetric line profiles are rare toward BGPS starless clump candidates in $\mathrm{HCO}^{+} 1-0$. We found no correlation between $\delta v$ and the physical parameters of the clumps, such as mass and virial parameter. Using HILL5 radiative transfer modeling of the best inflow candidates, we determine inflow velocities and mass inflow rates that range from 500 to $2000 M_{\odot} \mathrm{Myr}^{-1}$. At these accretion rates, the SCCs would double in mass over a freefall time. These clumps may be in a phase where they are embedded within a supersonic inflow and are growing in mass at a significant rate.

The $12 \mathrm{~m}$ ARO telescope has a beam size that is about the same size as or slightly larger than the clump radii we were observing. As a result, our six inflow candidates are only candidates for inflow motions. Higher spatial resolution mapping observations on telescopes such as the GBT and ALMA are necessary to confirm whether these objects are truly starless and whether they have global inflow motions.

We sincerely thank the staff and operators of the Arizona Radio Observatory. J.K.C., B.E.S., and Y.L.S. are supported in part by NSF grant AST-1410190. B.E.S. is also supported by the NSF Graduate Research Fellowship under grant no. DGE-
114395. J.K.C. thanks the Arizona NASA Space Grant Consortium for internship support.

Facility: ARO:12m.

Software: ds9, GILDAS CLASS.

\section{References}

Barnes, P. J., Yonekura, Y., Ryder, S. D., et al. 2010, MNRAS, 402, 73 Choi, M., Evans, N. J., II, Gregersen, E. M., \& Wang, Y. 1995, ApJ, 448, 742 De Vries, C. H., \& Myers, P. C. 2005, ApJ, 620, 800

Eden, D. J., Moore, T. J. T., Plume, R., et al. 2017, MNRAS, 469, 2163

Elia, D., Molinari, S., Schisano, E., et al. 2017, MNRAS, 471, 100

Ellsworth-Bowers, T. P., Rosolowsky, E., Glenn, J., et al. 2015, ApJ, 799, 29

Evans, N., II 2003, SFChem 2002: in Chemistry as a Diagnostic of Star Formation, ed. C. L. Curry \& M. Fich (Ottawa: NRC Press), 157

Evans, N. J., II, Lee, J.-E., Rawlings, J. M. C., \& Choi, M. 2005, ApJ, 626, 919

Feng, S., Beuther, H., Zhang, Q., et al. 2016, A\&A, 592, A21

Foreman-Mackey, D., Hogg, D. W., Lang, D., \& Goodman, J. 2013, PASP, 125,306

Fuller, G. A., Williams, S. J., \& Sridharan, T. K. 2005, A\&A, 442, 949

Ginsburg, A., Glenn, J., Rosolowsky, E., et al. 2013, ApJS, 208, 14

He, Y.-X., Zhou, J.-J., Esimbek, J., et al. 2015, MNRAS, 450, 1926

He, Y.-X., Zhou, J.-J., Esimbek, J., et al. 2016, MNRAS, 461, 2288

Hoq, S., Jackson, J. M., Foster, J. B., et al. 2013, ApJ, 777, 157

Jackson, J. M., Rathborne, J. M., Foster, J. B., et al. 2013, PASA, 30, e057

Jin, M., Lee, J.-E., Kim, K.-T., \& Evans, N. J., II 2016, ApJS, 225, 21

Kirk, H., Friesen, R. K., Pineda, J. E., et al. 2017, ApJ, 846, 144

Kirk, H., Myers, P. C., Bourke, T. L., et al. 2013, ApJ, 766, 115

Liu, X.-L., Xu, J.-L., Ning, C.-C., Zhang, C.-P., \& Liu, X.-T. 2017, arXiv:1709.08391

López-Sepulcre, A., Cesaroni, R., \& Walmsley, C. M. 2010, A\&A, 517, A66 Mangum, J. G. 1993, PASP, 105, 117

Mangum, J. G., \& Shirley, Y. L. 2015, PASP, 127, 266

Mardones, D., Myers, P. C., Tafalla, M., et al. 1997, ApJ, 489, 719

Matzner, C. D. 2017, arXiv:1712.01457

McKee, C. F., \& Ostriker, E. C. 2007, ARA\&A, 45, 565

Motte, F., Bontemps, S., \& Louvet, F. 2017, arXiv:1706.00118

Myers, P. C., Bachiller, R., Caselli, P., et al. 1995, ApJL, 449, L65

Myers, P. C., Mardones, D., Tafalla, M., Williams, J. P., \& Wilner, D. J. 1996 ApJL, 465, L133

Narayanan, G., Walker, C. K., \& Buckley, H. D. 1998, ApJ, 496, 292

Palmeirim, P., André, P., Kirk, J., et al. 2013, A\&A, 550, A38

Peretto, N., Fuller, G. A., Duarte-Cabral, A., et al. 2013, A\&A, 555, A112

Purcell, C. R., Balasubramanyam, R., Burton, M. G., et al. 2006, MNRAS, 367,553

Rygl, K. L. J., Wyrowski, F., Schuller, F., \& Menten, K. M. 2013, A\&A, 549, A5

Seo, Y. M., Hong, S. S., Lee, S. H., et al. 2011, ApJ, 736, 153

Shirley, Y. L. 2015, PASP, 127, 299

Shirley, Y. L., Ellsworth-Bowers, T. P., Svoboda, B., et al. 2013, ApJS, 209, 2

Smith, R. J., Hosokawa, T., Omukai, K., Glover, S. C. O., \& Klessen, R. S. 2012, MNRAS, 424, 457

Smith, R. J., Shetty, R., Beuther, H., Klessen, R. S., \& Bonnell, I. A. 2013, ApJ, 771, 24

Svoboda, B. E., Shirley, Y. L., Battersby, C., et al. 2016, ApJ, 822, 59

Tafalla, M., Mardones, D., Myers, P. C., et al. 1998, ApJ, 504, 900

Traficante, A., Fuller, G. A., Smith, R. J., et al. 2018, MNRAS, 473, 4975

Urquhart, J. S., Csengeri, T., Wyrowski, F., et al. 2014, A\&A, 568, A41

Wu, Y., Henkel, C., Xue, R., Guan, X., \& Miller, M. 2007, ApJL, 669, L37

Zhang, T., Wu, Y., Liu, T., \& Meng, F. 2016, ApJS, 224, 43 